\title{
Estimativa de Fluxo de Calor Bidimensional com Variação Temporal Empregando o Método de Monte Carlo com Cadeias de Markov
}

\author{
Gabriel Teixeira Soares das Neves ${ }^{1}$ \\ Instituto Federal de Educação, Ciência e Tecnologia Fluminense, IFF, Quissamã, RJ \\ Instituto Politécnico, UERJ, Nova Friburgo, RJ \\ Luiz Alberto da Silva Abreu ${ }^{2}$ \\ Diego Campos Knupp ${ }^{3}$ \\ Antônio José da Silva Neto ${ }^{4}$ \\ Instituto Politécnico, UERJ, Nova Friburgo, RJ
}

Resumo. Neste trabalho é abordado o problema inverso de estimativa de fluxo de calor bidimensional e com variação temporal aplicado a uma placa de alumínio. A formulação Classical Lumped foi empregada através da espessura da placa de modo a melhorar o custo computacional sem perda de qualidade da solução numérica do problema direto, calculada através do método de diferenças finitas. Uma abordagem Bayesiana, utilizando o Método de Monte Carlo com Cadeias de Markov, foi utilizada para a obtenção da solução do problema inverso. Para regularização do problema foi proposta uma priori na forma de Variação Total dos Parâmetros. A abordagem foi analisada utilizando medições simuladas e se mostrou robusta para casos com baixo ruído.

Palavras-chave. Problema Inverso, Estimação de Fluxo de Calor, Inferência Bayesiana, Variação Total dos Parâmetros

\section{Introdução}

Em um problema de transferência de calor nem sempre são conhecidos possíveis fluxos de calor no contorno e suas estimações a partir de medições indiretas configuram-se como um problema inverso [8]. Problemas desse tipo vêm se mostrando relevantes para aplicações em diversas áreas de interesse, como o superaquecimento de microchips em eletrônica [2], detecção de perda de calor na produção de energia [3] e a detecção de tumores na Medicina [5], para citar alguns. Técnicas como o método do gradiente conjugado já foram empregadas para a tarefa de estimar simultaneamente a variação espacial e temporal de fluxos de calor há algum tempo, como em [9] e [10].

\footnotetext{
${ }^{1}$ gabriel.neves@iff.edu.br

${ }^{2}$ luiz.abreu@iprj.uerj.br

${ }^{3}$ diegoknupp@iprj.uerj.br

4 ajsneto@iprj.uerj.br
} 
Recentemente, com a popularização de métodos estocásticos, abordagens Bayesianas, como o Método de Monte Carlo com Cadeias de Markov (MCMC) [6], na engenharia, problemas mais complexos e desafiadores puderam ser abordados. O avanço da tecnologia e a constante demanda por peças eletrônicas cada vez menores e, consequentemente, soluções em dissipação de calor, justifica a relevância de soluções na identificação e dissipação de fluxos pontuais de calor [4]. Embora o caso abordado em [8] seja similar ao apresentado aqui, este trabalho utiliza o Método de Monte Carlo com Cadeias de Markov para estimar o fluxo de calor aplicado a uma placa considerando duas dimensões espaciais e uma temporal, o que representa um significativo aumento nos parâmetros a serem estimados, com propostas de prioris na forma de total variation density para regularização do problema.

\section{Problema Direto}

Considere o problema de transferência de calor através de uma placa retangular plana, de dimensões $L_{x} \times L_{y} \times L_{z}$ sujeita à condição inicial $T(x, y, z, 0)=T_{\infty}$. Um fluxo de calor $q(x, y, t)$ é aplicado na superfície inferior $(z=0)$, enquanto a superfície superior $\left(z=L_{z}\right)$ é submetida a convecção natural com um fluido na temperatura ambiente $\left(T_{\infty}\right)$, como apresentado na figura 1. Essa situação é modelada através da equação de condução do calor e condições iniciais e de contorno dadas por [1].

$$
\rho c_{p} \frac{\partial T(\mathbf{X}, t)}{\partial t}=\frac{\partial}{\partial x}\left(k \frac{\partial T}{\partial x}\right)+\frac{\partial}{\partial y}\left(k \frac{\partial T}{\partial y}\right)+\frac{\partial}{\partial z}\left(k \frac{\partial T}{\partial z}\right)
$$

e

$$
\begin{array}{r}
k \frac{\partial T(\mathbf{X}, t)}{\partial \mathbf{n}}=q(x, y, t), z=0 \\
k \frac{\partial T(\mathbf{X}, t)}{\partial \mathbf{n}}=h\left(T_{\infty}-T(\mathbf{X}, t)\right), z=L_{z} \\
\frac{\partial T(\mathbf{X}, t)}{\partial \mathbf{n}}=0, x=0, x=L_{x}, y=0, y=L_{y} \\
T(X, 0)=T_{\infty}
\end{array}
$$

com

$$
0 \leq x \leq L_{x}, 0 \leq y \leq L_{y}, 0 \leq z \leq L_{z}, t \geq 0
$$

e onde $\mathbf{X}=(x, y, z), \rho$ é a massa específica $\left(k g / m^{3}\right), c_{p}$ é o calor específico do material $(\mathrm{J} /(\mathrm{kg}$. $K))$, e $k$ a condutividade térmica $(W /(m \cdot K))$.

Se o número de Biot, como definido em [1], calculado na direção $z$ for suficientemente pequeno, pode-se aplicar a técnica Classical Lumped através de $L_{z}$, que considera a temperatura constante e igual à média das temperaturas nesta direção. Além disso, utilizadas as condições de contorno $2 \mathrm{a}$ e $2 \mathrm{~b}$, é possível reescrever a equação $1 \mathrm{com}$ as respectivas condições de contorno e inicial, como apresentado a seguir.

$$
\rho c_{p} \frac{\partial T}{\partial t}=k\left(\frac{\partial^{2} T}{\partial x^{2}}+\frac{\partial^{2} T}{\partial y^{2}}\right)+\frac{q}{L_{z}}+\frac{h}{L_{z}}\left(T-T_{\infty}\right)
$$




$$
\begin{array}{r}
\frac{\partial T(\mathbf{X}, t)}{\partial \mathbf{n}}=0, x=0, x=L_{x}, y=0, y=L_{y} ; \\
T(\mathbf{X}, 0)=T_{\infty}
\end{array}
$$

com $0 \leq x \leq L_{x}, 0 \leq y \leq L_{y}, 0 \leq z \leq L_{z}, t \geq 0$ e, dessa vez, $\mathbf{X}=(x, y)$

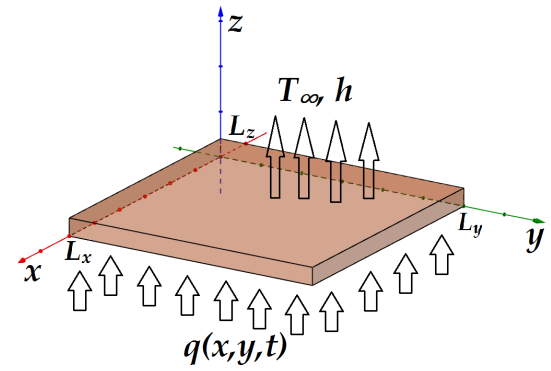

Figura 1: Representação do problema físico.

Para a solução do problema direto é utilizado um esquema de diferenças finitas sobre uma malha com $N_{x}$ pontos na direção $x, N_{y}$ pontos na direção $y$ e $N_{t}$ passos temporais. A convergência da solução do método foi verificada com a solução gerada por rotina intrínseca do MatLab ${ }^{\circledR}$.

\section{Problema Inverso}

Para a formulação do problema inverso são considerados dados experimentais simulados adicionando ruído à solução convergida do problema direto sobre uma malha $N_{x} \times N_{y} \times N_{t}$, fornecendo um total de $D=N_{x} \times N_{y} \times N_{t}$ valores experimentais. O vetor de dados experimentais pode ser expresso como

$$
\mathbf{Y}^{T}=\left(Y_{1}, Y_{2}, \ldots, Y_{D}\right)
$$

onde $Y_{i}, i=1,2 \ldots, D$, corresponde à medição em cada posição da malha.

O fluxo de calor $q(x, y, t)$ é estimado sobre uma malha com $D P=N P_{x} \times N P_{y} \times N P_{t}$ nós, levando a $D P$ parâmetros a serem estimados, $q_{i, j}^{k}, i=1,2, \ldots, N P_{x}, j=1,2, \ldots N P_{y}$ e $k=1,2, \ldots, N P_{t}$, correspondentes a $q\left(x_{i}, y_{j}, t_{k}\right)$. Então, o vetor de estimativas para os parâmetros procurados pode ser escrito como

$$
\mathbf{P}^{T}=\left(q_{1}, q_{2}, \ldots, q_{D P}\right)
$$

Para a solução do problema inverso é utilizada uma abordagem Bayesiana, pois possibilita combinar informações a priori no cálculo das estimativas através do teorema de Bayes [6], característica importante para regularização de problemas em que muitos parâmetros são estimados. Nesse caso, a solução do problema consiste numa curva de densidade com a probabilidade a posteriori, expressa por 


$$
\pi_{\text {post }}(\mathbf{P})=\pi(\mathbf{P} \mid \mathbf{Y})=\frac{\pi_{\text {prior }}(\mathbf{P}) \pi(\mathbf{Y} \mid \mathbf{P})}{\pi(\mathbf{Y})}
$$

onde $\pi_{\text {post }}(\mathbf{P})$ é a distribuição posterior de probabilidade dos parâmetros $\mathbf{P}, \pi_{\text {prior }}(\mathbf{P})$ é a distribuição de probabilidades dos dados a priori, $\pi(\mathbf{Y})$ a distribuição marginal de probabilidade dos dados experimentais, que desempenha papel de constante de normalização e $\pi(\mathbf{Y} \mid \mathbf{P})$, a função de verossimilhança, expressa analiticamente na forma

$$
\pi(\mathbf{Y} \mid \mathbf{P})=(2 \pi)^{-D / 2}|W|^{-1 / 2} \times \exp \left[-\frac{1}{2}[\mathbf{Y}-T(\mathbf{P})]^{t} W^{-1}[\mathbf{Y}-T(\mathbf{P})]\right]
$$

onde $D$ é a quantidade de medidas, $W$ é a matriz de covarianças dos erros das medidas e $T(\mathbf{P})$ é o vetor contendo a solução do problema direto dados os valores de $\mathbf{P}$, nas mesmas posições que as medidas experimentais.

É utilizado o algoritmo Metropolis-Hasting, descrito detalhadamente em [6], para gerar candidatos para a distribuição a posteriori. A implementação se inicia com a seleção de uma densidade de movimentação $p\left(\mathbf{P}^{*}, \mathbf{P}^{(t-1)}\right)$, que é utilizada para gerar um candidato $\left(\mathbf{P}^{*}\right)$ para o novo estado da cadeia, dado o estado anterior $\mathbf{P}^{(t-1)}$. Criando assim a sequência $\left\{\mathbf{P}^{1}, \mathbf{P}^{2}, \ldots, \mathbf{P}^{n}\right\}$, que deve convergir para a solução procurada. Note que os primeiros termos, ainda não convergidos, devem ser ignorados.

Para modelar a priori, é empregada a abordagem total variation density [7], dada na forma

$$
\pi(\mathbf{P}) \propto \exp \left[-\frac{\gamma}{2} T V(\mathbf{P})\right]
$$

com o parâmetro de regularização $(\gamma)$ escolhido empiricamente para o problema e onde, para o caso estudado,

$$
\begin{aligned}
T V(\mathbf{P}) & =\sum_{i=2}^{N P_{x}-1} \sum_{j=2}^{N P_{y}-1} \sum_{k=2}^{N P_{t}-1} \Delta x\left[\left|q_{i, j}^{k}-q_{i+1, j}^{k}\right|+\left|q_{i, j}^{k}-q_{i-1, j}^{k}\right|\right]+ \\
& +\Delta y\left[\left|q_{i, j}^{k}-q_{i, j+1}^{k}\right|+\left|q_{i, j}^{k}-q_{i, j-1}^{k}\right|\right]+\Delta t\left[\left|q_{i, j}^{k}-q_{i, j}^{k+1}\right|+\left|q_{i, j}^{k}-q_{i, j}^{k-1}\right|\right]
\end{aligned}
$$

\section{Resultados e Discussões}

Foi considerado o caso de uma placa retângular com dimensões $0,08 m \times 0,04 m$ e 0,003m de espessura, e duração de 50 segundos do fenômeno de condução de calor. As propriedades do material simulado são próximas dos utilizados na manufatura de chips eletrônicos, com $k=0,2 \mathrm{~W} / \mathrm{mK}$ e $\rho c_{p}=9,6 \times 10^{5} \mathrm{~J} / \mathrm{m}^{3} \mathrm{~K}$. O coeficiente de transferência de calor escolhido foi de $h=12 \mathrm{~W} / \mathrm{m}^{2} \mathrm{~K}$, com $T_{\infty}=20^{\circ} \mathrm{C}$. O fluxo, em $\mathrm{W} / \mathrm{m}^{2}$, que pretende-se estimar é o descrito pela equação 11, que consiste em uma função com variações abruptas no tempo e espaço.

$$
q(x, y, t)=\left\{\begin{array}{cc}
500 & 0,02 \leq x \leq 0,04,0 \leq y \leq 0,02,20 \leq t \leq 40 \\
0 & 0,02 \geq x \geq 0,04 \text { ou } 0 \geq y \geq 0,02 \text { ou } 20 \geq t \geq 40
\end{array}\right.
$$


A malha utilizada para estimação do fluxo possui $9 \times 9 \times 11$ nós, o que leva a 891 parâmetros a serem estimados, $q_{i j}^{k}$, com $i, j=1,2, \ldots, 9$ e $k=1,2, \ldots, 11$ e os dados experimentais foram simulados adicionando um ruído com distribuição Gaussiana, média zero e desvio padrão $\sigma=0.05^{\circ} \mathrm{C}$ à solução convergida do problema direto calculada via diferenças finitas. As propostas para as estimativas do fluxo de calor a serem utilizadas no MCMC são distribuições normais com desvio padrão $\delta=5 \mathrm{~W} / \mathrm{m}^{2}$. Foram calculados 50000 estados na cadeia de Markov, sendo os 10000 primeiros descartados [6].

Devido ao elevado número de parâmetros a serem determinados, faz-se necessário o uso de técnicas de regularização para o problema, na forma de informação a priori. Os resultados aqui apresentados comparam os casos em que $\gamma=0$ (sem priori), $\gamma=0,0001 \mathrm{e}$ $\gamma=1$. A figura 2 apresenta o fluxo estimado em cada caso em comparação com o valor exato na direção $x, \operatorname{com} y=0,01 m$ e $t=30 s$ (figura 2(a)), na direção $y, \operatorname{com} x=0,02 m$ e $t=30 s$ (figura 2(b)), e a evolução no tempo, com $x=0,02 s$ e $y=0,01 s$ (figura 2(c)). Os resultados para o caso sem priori apresentam comportamento bastante irregular, enquanto os casos em que utiliza a priori do tipo total variation aproximam com boa concordância o fluxo exato, o que demonstra o bom desempenho na obtenção de estimativas para fluxos de calor que apresentem variações bruscas no tempo e espaço.

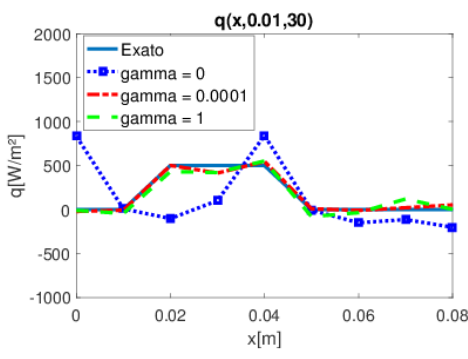

(a)

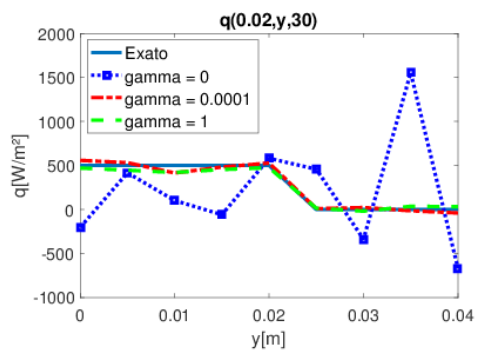

(b)

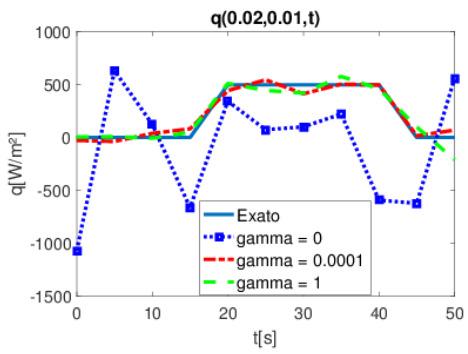

(c)

Figura 2: Comparativo entre fluxo estimado e exato nas direções $x$ (a), $y$ (b) e no tempo (c).

Para a análise do erro na estimativa do fluxo de calor é utilizado o erro RMS, como definido a seguir para a aproximação de um conjunto de parâmetros $\epsilon_{i, j}^{k}$ calculados em relação a valores exatos $\epsilon\left(x_{i}, y_{j}, t_{k}\right)$ numa malha $N_{x} \times N_{y} \times N_{t}$, e que será aplicado para o fluxo e para a temperatura calculada com o modelo após a solução do problema inverso. Os resultados são apresentados na tabela 1 . 
$R M S_{\epsilon}=\sqrt{\frac{1}{N_{x}} \frac{1}{N_{y}} \frac{1}{N_{t}} \sum_{i=1}^{N_{x}} \sum_{j=1}^{N_{y}} \sum_{k=1}^{N_{t}}\left[\epsilon\left(x_{i}, y_{j}, t_{k}\right)-\epsilon_{i, j}^{k}\right]^{2}}$

Tabela 1: Erros calculados para diferentes valores de $\gamma$.

\begin{tabular}{|c|c|c|c|}
\hline Erro & $\gamma=0$ & $\gamma=0,0001$ & $\gamma=1$ \\
\hline$R M S_{q}$ & 9,9769 & 2,8646 & 0,0255 \\
\hline$R M S_{T}$ & 0,0065 & 0,0011 & 0,00061513 \\
\hline
\end{tabular}

Observando os valores dos erros RMS para cada caso fica bastante claro como a modelagem das prioris na forma de total variation cumpre o papel de regularizar o problema de forma satisfatória. Mesmo com pouca influência, $\operatorname{com} \gamma=0,0001$, já é possível notar uma diminuição significativa no erro calculado. Quando se considera $\gamma=1$ os erros calculados diminuem de forma expressiva. A figura 3 apresenta a reconstrução do fluxo encontrado para o tempo $t=30 \mathrm{~s}$ (figura 3(a)) e o resíduo entre a temperatura exata e a estimada em $t=50 \mathrm{~s}$ (figura 3(b)), com $\gamma=1 \mathrm{em}$ ambos os casos. O baixo valor do resíduo confirma a a qualidade das estimativas obtidas com a metodologia desenvolvida.

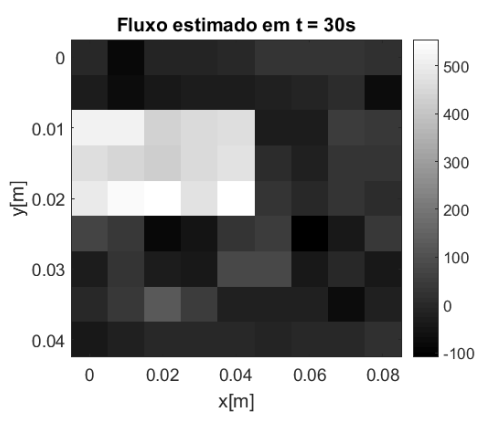

(a)

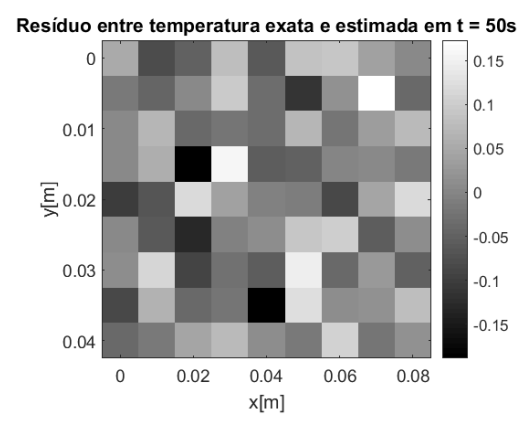

(b)

Figura 3: (a) Fluxo estimado $\left(\mathrm{W} / \mathrm{m}^{2}\right)$ e (b) resíduo entre temperaturas estimadas e medidas $\left(\mathrm{C}^{o}\right)$ para $\gamma=1$.

\section{Conclusões}

Este trabalho tratou do problema inverso de estimativa do fluxo de calor em meios bidimensionais com variação temporal, utilizando o Método de Monte Carlo com Cadeias de Markov e a priori total variation. Os resultados da estimativa para o fluxo e para o perfil de temperaturas apresentam boa concordância com o fluxo de calor imposto, determinado com o problema direto, e os dados experimentais para a temperatura utilizados no problema inverso, respectivamente. 


\section{Agradecimentos}

Os autores agradecem o suporte financeiro da FAPERJ, do CNPq e da CAPES. O primeiro autor agradece o suporte financeiro do IFF, instituição na qual é professor.

\section{Referências}

[1] T. L. Bergman, A. S. Lavine, F. P Incropera e D. P. DeWitt, Fundamentals of Heat and Mass Transfer, John Wiley \& Sons, USA, 2011.

[2] W. L. Chen e Y. C. Yang. Estimation of the Transient Heat Transfer Rate at the Boundary of an Electronic Chip Packaging, Numerical Heat Transfer, Part A: Applications, volume 54, 10:945-961, 2008. DOI: 10.1080/10407780802473574.

[3] S. Govert. Heat Loss Prediction of a Confined Premixed Jet Flame Using a Conjugate Heat Transfer Approach, International Journal of Heat and Mass Transfer, 2016. DOI: $10.1016 /$ j.ijheatmasstransfer.2016.10.122.

[4] G. Hetsroni, A. Mosyak e Z. Segal. Nonuniform Temperature Distribution in Electronic Devices Cooled by Flow in Parallel Microchannels, IEEE Transcations on Components and Packaging Technologies, volume 24, 16-23, 2001. DOI: 10.1109/6144.910797.

[5] A. Jafarimoghaddam e S. Aberoumand. A Bayesian Approach and Total Variation Priors in 3D Electrical Impedance Tomography, Engineering Science and Technology, an International Journal, 2016. DOI: 10.1109/IEMBS.1998.745625.

[6] J. Kaipio e E. Somersalo, Statistical and Computational Inverse Problems, Springer, USA, 2004.

[7] M. F. Mescolin, L. A. da S. Abreu, D. C. Knupp e A. J. Silva Neto, Estimation of Spatially and Time Varying Heat Flux via Markov chain Monte Carlo Method and Integral Transforms, Procedings of ENCIT, 2016.

[8] H. R. B. Orlande. Inverse Problems in Heat Transfer: New Trends on Solution Methodologies and Applications, Journal of Heat Transfer, volume 134, 2012. DOI: 10.1115/1.4005131.

[9] A. J. Silva Neto e M. N. Özisik, Simultaneous Estimation of Location and TimewiseVarying Strength of a Plane Heat Source, Numerical Heat Transfer, Part A: Applications, volume 24, 4:467-477, 1993. DOI: 10.1080/10407789308902635.

[10] J. Su e A. J. Silva Neto, Two-Dimensional Inverse Heat Conduction Problem of Source Strength Estimation in Cylindrical Rods, Applied Mathematical Modelling, volume 25, 10:861-872, 2001. DOI: 10.1016/S0307-904X(01)00018-X. 